\title{
Microfluidic Fabrication and Micromechanics of Permeable and Impermeable Elastomeric Microbubbles
}

\author{
Wynter J. Duncanson, ${ }^{*}, \dagger, \S$ Thomas E. Kodger, ${ }^{\dagger, \S}$ Sahab Babaee, ${ }^{\dagger}$ Grant Gonzalez, $^{\dagger}$ David A. Weitz, ${ }^{\dagger}$ \\ and Katia Bertoldi ${ }^{\dagger}$ \\ ${ }^{\dagger}$ School of Engineering and Applied Sciences Cambridge, Harvard University, Cambridge, Massachusetts 02138, United States
${ }^{\ddagger}$ Department of Chemical Engineering, Nazarbayev University, Astana, 010000, Kazakhstan
}

ABSTRACT: We use droplet microfluidics to produce monodisperse elastomeric microbubbles consisting of gas encapsulated in a polydimethylsiloxane shell. These microbubbles withstand large, repeated deformations without rupture. We perform $\mu \mathrm{N}$-scale compression tests on individual microbubbles and find their response to be highly dependent on the shell permeability; during deformation, the pressure inside impermeable microbubbles increases, resulting in an
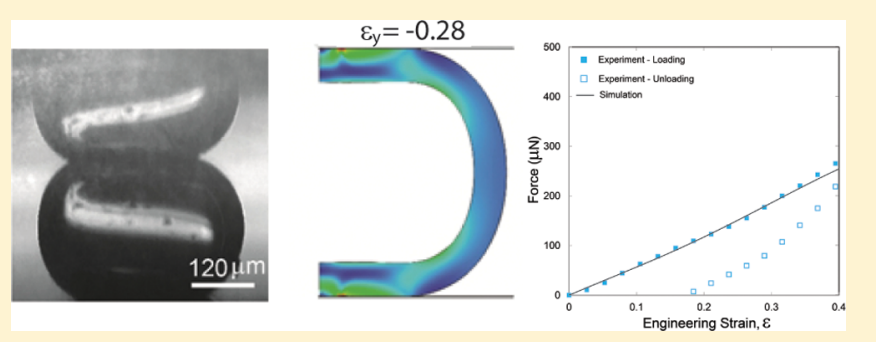
exponential increase in the applied force. Finite element models are used to interpret and extend these experimental results enabling the design and development of deformable microbubbles with a predictable mechanical response. Such microbubbles can be designed to repeatedly transit through the narrow constrictions found in a porous medium functioning as probes of the local pressure.

\section{INTRODUCTION}

Microbubbles are spherical particles consisting of gas encapsulated in a shell. They are conventionally used as components in aerated food products, materials for energy storage and wastewater treatment, vehicles for drug delivery, and tools for contrast enhancement in ultrasound. ${ }^{1-3}$ In addition, microbubbles are used as pressure probes as their size change registers as a shift in the nuclear magnetic resonance signal intensity; this ability to dynamically change size enables their use as local pressure mapping agents to study fluid flow in porous media ${ }^{4,5}$ and to map local pressure changes in oil reservoirs. To truly be useful in such confined geometries, microbubbles must be able to reversibly deform following their passage through confined porous space under high pressure conditions. Thus, a microbubble shell with the combined mechanical properties of high flexibility and resilience is required.

The mechanical properties of the microbubble shell can be altered by changing two aspects: physical thickness and chemical composition. Typically, shells are chemically composed of either lipids, which are flexible yet mechanically weak, or polymers, which tend to be rigid yet mechanically strong. ${ }^{6}$ Furthermore, increases in shell thickness always result in higher bending stiffness. Modification of such physiochemical characteristics is not easily attained through conventional microbubble fabrication strategies. To circumvent these limitation, the versatility of droplet microfluidics can be used to control the physical properties of microbubble size and shell thickness by flow rate variations and the chemical composition by the flexibility in choice of material. ${ }^{7-10}$ In this way, shells that are both flexible and mechanically resilient can be produced from elastomeric polydimethylsiloxane (PDMS), ${ }^{7}$ whose stiffness can be adjusted in bulk by altering the ratio of the two-parts, base and cross-linker, during the hydrosilylation reaction. Further tuning of these physiochemical properties of microbubbles may increase their utility and robustness.

Once fabricated, a number of available experimental techniques may be used to measure the mechanical properties of microbubbles. ${ }^{11-16}$ One such technique, parallel plate squeezing, directly measures the force required to squeeze spheres or shells between two rigid parallel plates while measuring the distance, or gap, between the plates. ${ }^{15}$ Moreover, numerical simulations can be used to both substantiate the experimental tests and to a priori estimate the deformation extents of the microbubbles. Despite the availability of experimental compressive and numerical analysis techniques, their use in conjunction to elicit variations in engineered microbubbles fabricated through controllable methods such as microfluidics has not been achieved.

In this paper, we produce flexible and resilient microbubbles and elucidate their response to mechanical deformation. We use droplet microfluidics to prepare monodisperse elastomeric microbubbles with a UV-curable PDMS shell. We measure the deformability of these microbubbles by conducting mechanical compression tests on individual microbubbles to obtain forcedisplacement curves which we then compare to finite element models. Remarkably, this combination of numerical and experimental tools enables the design and development of deformable microbubbles with a predictable mechanical response.

Received: December 12, 2014

Revised: February 23, 2015

Published: March 2, 2015 


\section{FABRICATION}

We generate elastomer microbubbles using a glass capillary microfluidic device: A cylindrical injection capillary and a collection capillary are coaxially aligned within a square capillary. ${ }^{17}$ We use pressure-driven flow to drive a nitrogen gas stream into the injection capillary, and a syringe pump to drive the flow of the middle phase consisting of a PDMS monomer in the space between the square and injection capillaries; this yields the primary gas-in-oil emulsion. To obtain the final gas-in-oil-in-water double-emulsion, the primary emulsion is further emulsified by the syringe pump-driven continuous phase, as shown in the schematic and optical image in Figure 1A. Because of the high viscosity of the middle oil
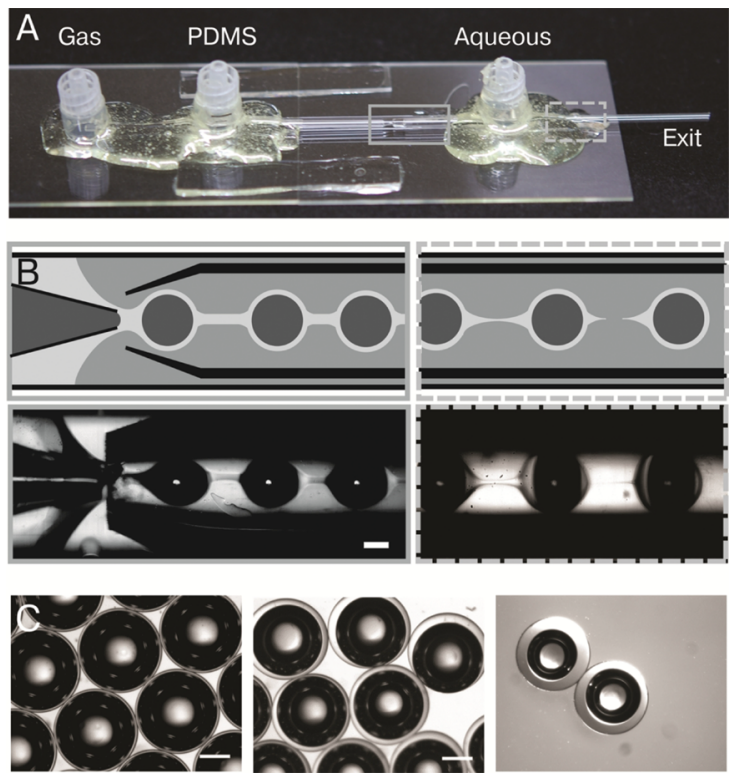

Figure 1. (A) Glass microfluidic device with the location of the droplet production area highlighted by a solid border and the downstream region highlighted by a dashed border. (B) Top panels: schematic illustration of the microuidic droplet generation of bubbles stabilized by an unpolymerized PDMS shell at the orifice, which further downstream breaks into individual drops. Bottom panels: experimental optical microscope images showing the emulsification process. (C) Optical image of three monodispersed microbubble populations formed using a PDMS flow rate of $1.0,1.5$, and $2.0 \mathrm{~mL} / \mathrm{h}$, from left to right. Scale bar is $200 \mu \mathrm{m}$ for all images.

phase, breakup occurs downstream of the orifice where the fluids intersect in the device (Figure 1B). These emulsions are exposed to UV $(265 \mathrm{~nm})$ at the device outlet thereby polymerizing the PDMS monomer shell, yielding elastomer microbubbles. Through careful changes to the middle phase flow rate from $1,1.5$, to $2.0 \mathrm{~mL} / \mathrm{h}$, we obtain three distinct shell thicknesses, as shown in Fig. $1 \mathrm{C}$.

PDMS elastomers are advantageous being both chemically inert and mechanically stable up to $200{ }^{\circ} \mathrm{C}$, while also highly permeable to most gases. For applications such as enhanced oil recovery, a microbubble is forced through a confined geometry, its volume decreases, and the interior pressure increases; allowing gases to permeate the shell may relieve this surface stress. Therefore, we fabricate both impermeable and permeable microbubbles by choosing a continuous phase during fabrication of either $20 \mathrm{wt} \%$ poly(vinyl alcohol) (PVA, $87-89 \%$ hydrolyzed) or 10 wt \% polyethylene glycol (PEG) with sodium dodecyl sulfate (SDS) as surfactant. Partially hydrolyzed poly(vinyl alcohol), PVA, is used as a polymeric surfactant in microfluidic experiments as it provides not only interface stability but also a high shear stress due to its increased viscosity over dilute surfactant solutions. ${ }^{18}$ However, PVA is also an excellent film former; ${ }^{19}$ even after many washings with water, a residual surface film is nearly always present. $^{20,21}$ It is this surface film that inhibits the gases from permeating the PDMS shell. By contrast, a solution of PEG and SDS, increases viscosity and interface stability, respectively, but does not irreversibly adsorb to the surface; thus permitting gases to transport through the PDMS shell.

\section{MECHANICAL TESTING}

To evaluate the mechanical properties of the these microbubbles, we use a parallel plate-plate compression apparatus constructed of two silicon wafers, an analytical balance and a linear translation motor, as shown in the schematic in Fig. 2A.
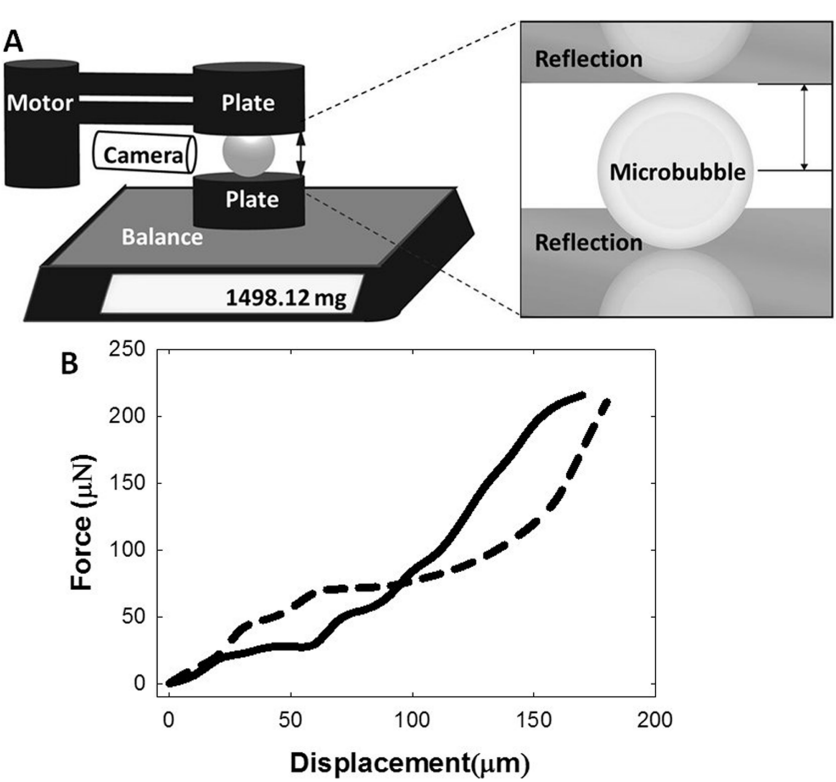

Figure 2. (A) Experimental setup of the parallel plate compression test. (B) Typical loading (solid) and unloading (dashed) curves for an impermeable microbubble under compression.

A single microbubble is placed on a silicon wafer that is affixed to the plate of the analytical balance; a precision of $0.1 \mathrm{mg}$ enables measurement of forces below $10^{-6} \mathrm{~N}$. A silicon wafer affixed to the linear motor serves as the top plate. We program the linear motor to make predetermined displacement steps, applying a fixed strain to the microbubble while measuring the balance readout. To conduct a force-displacement measurement, the point of first contact between the microbubble and top plate is set as the zero-displacement. Due to the reflectivity of silicon, the reflection of the microbubble is seen on both the top and bottom plates, as shown in the second panel of Fig. 2A. The top plate is lowered to its first position, and the microbubble deforms; after adequate relaxation time, the compression cycle continues. At maximal compression, strain $\sim 70 \%$, the decompression cycle begins. In a typical force versus displacement curve for the compression of an impermeable microbubble, the loading and unloading curves do not overlap. There is, however, an indication of complete mechanical recovery in which the curves do not exhibit 
hysteresis as both curves return to zero force at zero displacement, as shown in Fig. 2B. This recovery highlights that the PVA coating serves as an effective barrier to the gas contained within the microcavity. The slope of the loading curve is low, $\sim 0.5$, up to a displacement of $60 \mu \mathrm{m}$ and increases to 1.7 until loading is complete, indicating that less force is required to make the initial deformations. At more significant deformations, greater force is required, due to an increase in the internal pressure within the impermeable microbubble. By contrast, from the point of maximum displacement, the unloading curve begins with a high negative slope of -0.9 to $160 \mu \mathrm{m}$ and decreases to a lower slope of -0.6 until $60 \mu \mathrm{m}$. Notwithstanding these differences in the shapes of the curves, the area under each curve remains the same, demonstrating equivalent work is required for compression and decompression. These deformation experiments are repeated for permeable microbubbles.

\section{NUMERICAL SIMULATIONS}

To elucidate the deformation curves obtained experimentally, we numerically investigate the deformation of the microbubbles by using the commercial finite element software Abaqus/ Standard. 2D axisymmetric models are meshed with approximately 1500 four-node bilinear axisymmetric quadrilateral elements with reduced integration (element type CAX4R in Abaqus), and the accuracy of each mesh is ascertained through a mesh refinement study. The response of the microbubble is captured by using a nearly-incompressible Neo-Hookean model $^{22}$ (with Poisson's ratio $\nu_{0}=0.49$ ), whose strain energy density is given by

$$
U=\frac{G_{0}}{2}\left(\bar{I}_{1}-3\right)+\frac{K_{0}}{2}(J-1)
$$

where $\bar{I}_{1}=J^{-2 / 3} \operatorname{tr}\left[\operatorname{dev}\left(F^{T} F\right)\right], J=\operatorname{det}(F)$ and $F$ is the deformation gradient. Moreover, $G_{0}$ and $K_{0}$ are the shear and bulk modulus in the undeformed configuration and they are obtained by fitting the response under uniaxial compression of the bulk materials, resulting in $\mathrm{G}_{0}^{\operatorname{Imp}}=40 \mathrm{kPa}, \mathrm{K}_{0}^{\operatorname{Imp}}=1987 \mathrm{kPa}$, and $\mathrm{G}_{0}^{P}=60 \mathrm{kPa}, \mathrm{K}_{0}^{P}=2980 \mathrm{kPa}$ for impermeable and permeable bubbles, respectively.

To accurately simulate the microbubble response, we account for the pressure on the shell that is exerted by the contained gas using the surface-based fluid cavity capability in Abaqus. Particularly for the permeable microbubbles, we assume that the gas in the core is in equilibrium with the surrounding environment and at atmospheric pressure. By contrast, for the impermeable microbubbles, we account for the fact that the gas inside the cavity may be at a higher pressure than the surrounding environment by considering initial pressures ranging from 1 to $1.5 \mathrm{~atm}$. Moreover, we treat the gas inside the cavity as an ideal gas, so that $p V=$ constant, where $p$ and $V$ denote the pressure and volume of the gas, respectively.

\section{RESULTS AND DISCUSSION}

We compare the experimental and numerical responses obtained from compression tests of both the permeable and impermeable microbubbles. In Figure 3, we report experimental and numerical snapshots of the impermeable (top) and permeable (bottom) microbubbles at different levels of applied compression. Interestingly, the numerical results reveal significant differences in the stress magnitude and spatial

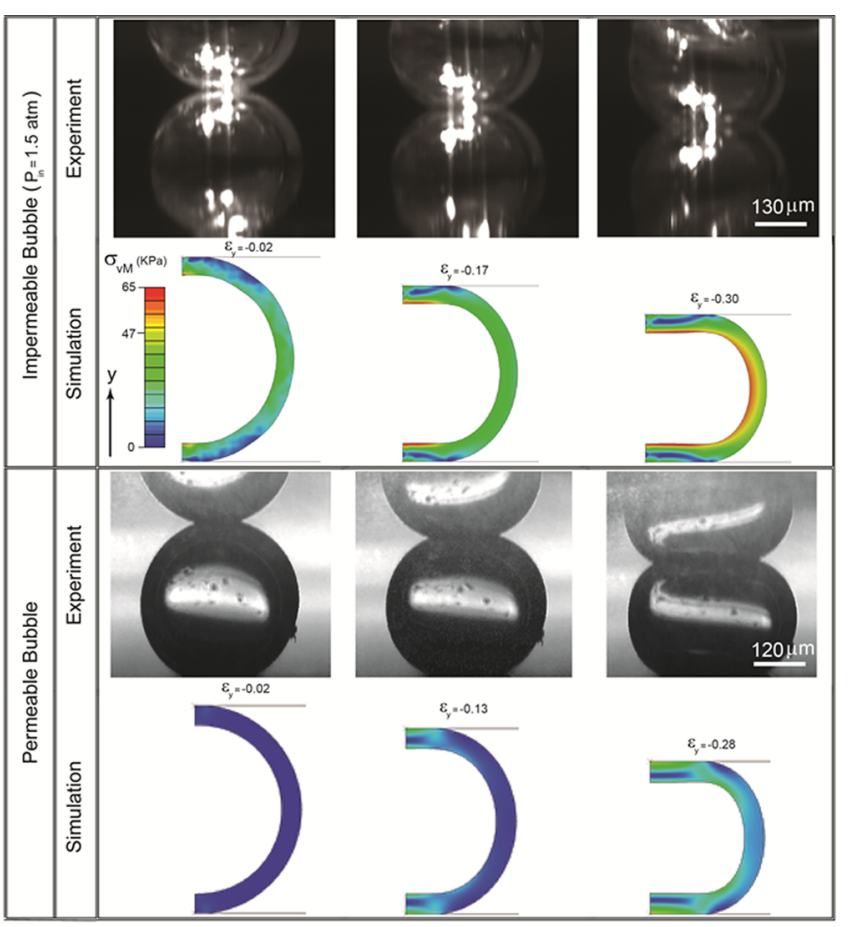

Figure 3. A comparison of the experiment and simulation results of the impermeable and permeable microbubbles. In the top panel, an impermeable bubble with outer diameter $D_{\text {out }}=446 \mu \mathrm{m}$ and inner diameter $D_{\text {in }}=365 \mu \mathrm{m}$ is uniaxially compressed to a strain $=-0.30$. The corresponding stress distribution diagram shows a maximal von Mises stress of $65 \mathrm{kPa}$. In the bottom panel, a permeable bubble $\left(D_{\text {out }}\right.$ $=380 \mu \mathrm{m}$ and $\left.D_{\text {in }}=310 \mu \mathrm{m}\right)$ is compressed to a strain $=-0.28$ with a corresponding stress distribution diagram showing a maximal von Mises stress of $47 \mathrm{kPa}$.

distribution for the case of permeable and impermeable microbubbles upon compression. For the impermeable bubble a maximum von Mises stress of $65 \mathrm{kPa}$ is observed when the shell is compressed to a strain of $30 \%$, while for the permeable bubble a smaller maximum von Mises stress of $47 \mathrm{kPa}$ is found despite the fact that the shear modulus of the material is larger, $\mathrm{G}_{0}^{\mathrm{Imp}}<\mathrm{G}_{0}^{\mathrm{P}}$. Moreover, in the impermeable bubble the maximum stress occurs at the interface with the cavity as a result of the increasing pressure of the gas, while in the permeable case it is located at the point of contact with the top and bottom plates.

We now quantitatively compare the experimental forcestrain curves with those of the simulations. The force versus engineering strain, $\epsilon$, loading curves for a impermeable bubble are shown in Figure 4A. The markers correspond to the experimental data, while the results of numerical simulations with different values of initial pressure for the gas inside the cavity are reported as continuous lines. We find that the closest fit to the experimental data occurs for an initial cavity pressure of $1.5 \mathrm{~atm}$. The agreement between numerical and experimental data is excellent up to $\epsilon=0.2$, (vertical dashed line in Figure $4 \mathrm{~A}$ ), wherein the simulation begins to deviate. Moreover, the nonlinear force-strain curves for the impermeable microbubble further confirm the influence of the pressure that builds up inside the cavity on the mechanical properties and indicate that greater force is required to deform the microbubble shell after a critical strain value of $20 \%$, as shown in Figure 4A. By contrast, for the loading curve of the permeable bubble, the shape of the force-strain curve is linear up to $\epsilon=0.4$, which is also the limit of the finite element model as shown in Figure 4B. In addition, 
A

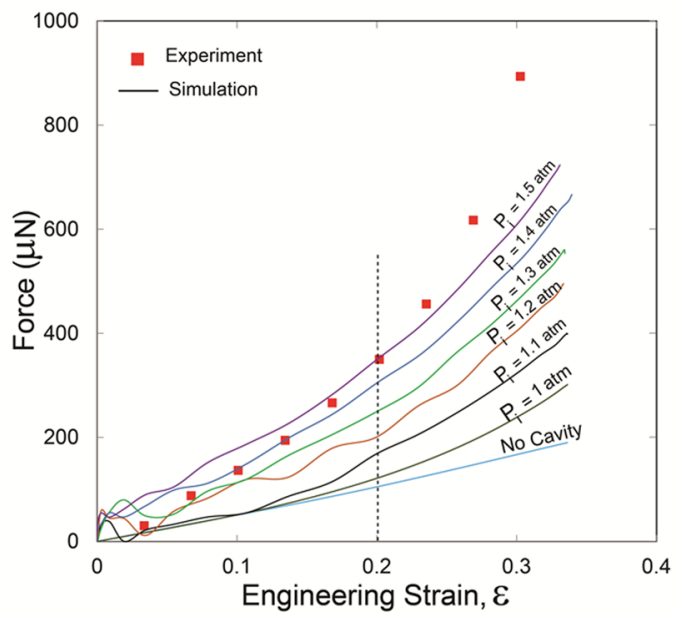

B

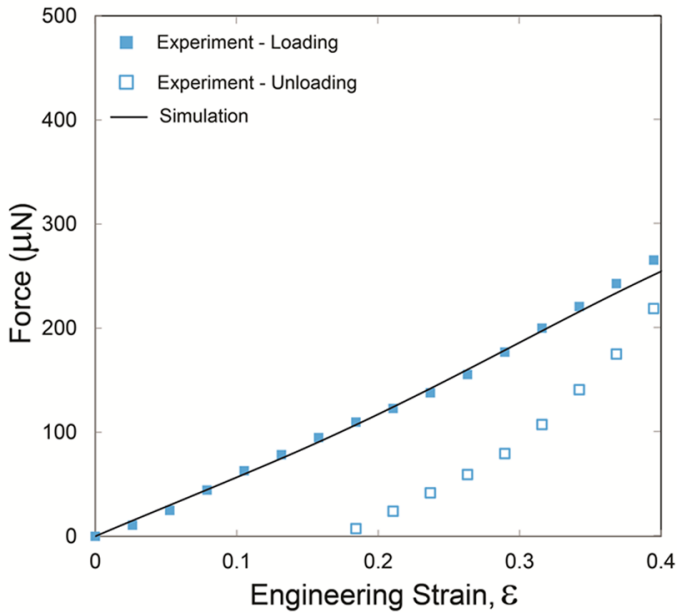

Figure 4. Force versus engineering strain, $\epsilon$, for impermeable (A) and permeable (B) microbubbles shown in Figure 3. The experimental data and simulation results are shown by markers and solid lines, respectively.

the loading and unloading curves show clear hysteresis as the unloading curve returns to zero force at $\epsilon=0.18$, whereas the force only returns to zero at $\epsilon=0$ for the impermeable microbubble as shown in Figure 2B.

Finally, we validate the mechanical resilience of these wellcharacterized microbubbles by passing them through constrictions that simulate the porous space of the end-use application: porous rock in oil reservoirs. To conduct this simple test, we pull a glass capillary to have a single smooth constriction into which one permeable microbubble is loaded. We connect the entrance of the capillary to a pressure source equipped with a pressure transducer; the exit is open to atmospheric pressure. Initially, at low pressure, the microbubble starts before the constriction, as shown in Figure 5A. As the

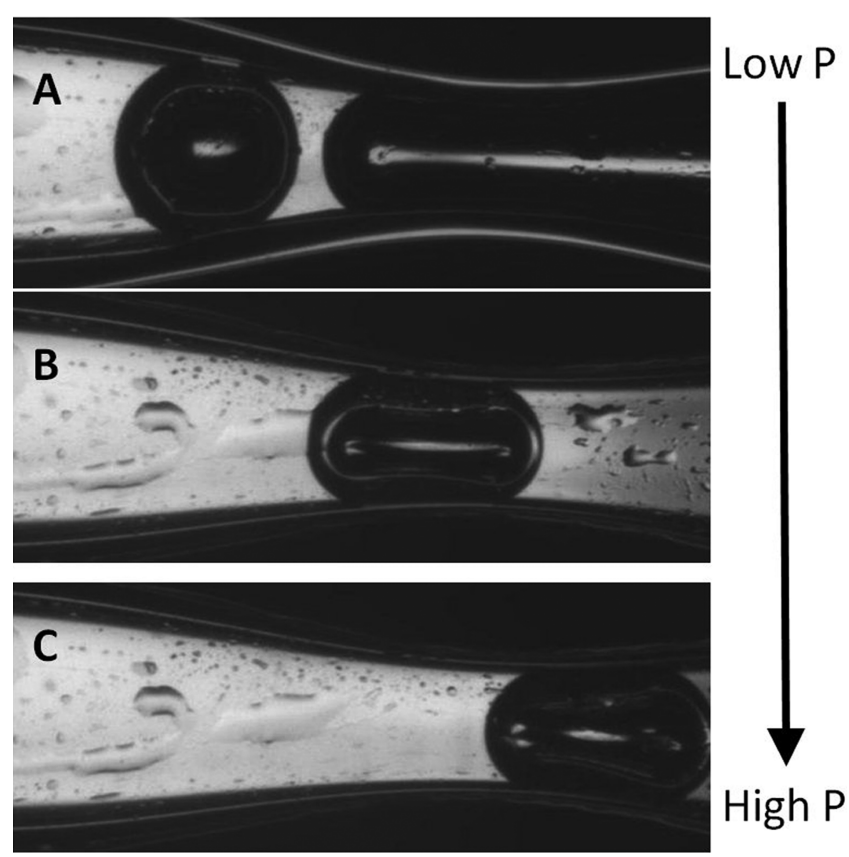

Figure 5. Stages of microbubble transit through a glass constriction. The position of the microbubble in panels $\mathrm{A}$ and $\mathrm{B}$ is at equilibrium with the applied pressure. The applied pressures are (A) $0.5 \mathrm{psi}$, (B) $4.5 \mathrm{psi}$, and (C) $6.1 \mathrm{psi}$. pressure increases, the microbubble is forced into the constriction and deforms to the size of the constriction, as shown in Figure 5B. As the microbubble exits the constriction, it begins to recover its original shape and flows out the exit of the capillary, as shown in Figure 5C. This simple test demonstrates elastomer microbubble resilience in response to applied pressure as it passes through a constriction.

\section{CONCLUSION}

We have used microfluidics to prepare PDMS elastomer microbubbles with controllable shell dimensions and permeability by varying the shell-coating. We directly measured their mechanical properties through the use of a $\mu \mathrm{N}$-scale parallel plate compression test, and used a finite element to interpret these experimental results. By inputting the shell modulus, dimensions, and permeability into this model, we can accurately predict the microbubble mechanical response. The shell thickness may be altered beyond the limits explored in this paper by changing the microfluidic flow geometry to form ultrathin shells. ${ }^{23}$ Likewise, the shell modulus may be made softer or stiffer by using other commercially available PDMS formulations ranging in stiffness from $\sim 2 \mathrm{MPa}$ to $\sim 1 \mathrm{kPa}$. This range of potential mechanical properties permits future controlled porous media experiments where microbubbles could be tailored to deform and passage through small pores or to clog high flow rate pores and increase fluidic resistance. The ability to use numerical models expands the possibilities of designing microfluidic microbubbles with predictable mechanical properties.

\section{EXPERIMENTAL SECTION}

All chemicals were purchased from Sigma-Aldrich and used as received unless otherwise noted. The external continuous phase consisted of either $10 \mathrm{mM}$ sodium dodecyl sulfate (SDS) and $10 \mathrm{wt} \%$ polyethylene glycol $(\mathrm{Mn} \approx 20000 \mathrm{~g} / \mathrm{mol})$ or $20 \mathrm{wt} \%$ poly(vinyl alcohol) $\left(M_{\mathrm{w}} \approx\right.$ 13000 to $23000 \mathrm{~g} / \mathrm{mol}, 87-89 \%$ hydrolyzed) in water which was bubbled with nitrogen for $15 \mathrm{~min}$ prior to use in the microfluidic device. The shell fluid is composed of a UV curable PDMS formulation: ${ }^{24} 1$ wt $\%$ of photoinitiator, 2,2-dimethoxy-2-phenylacetophenone, was dissolved into methacrylate-modified PDMS prepolymer, RMS-033 (Gelest). This mixture was bubbled with nitrogen for $15 \mathrm{~min}$ prior to use in the microfluidic device. The inner 
dispersed phase was a nitrogen source used at a fixed pressure of 15 psi.

The glass capillary microfluidic device was fabricated according to published methods. ${ }^{17}$ Square glass capillaries were purchased from Atlantic International Technologies. Round glass capillaries were purchased from World Precision Instruments and tapered using a Sutter P-97 capillary puller. For the constriction experiments, a $1 \mathrm{~mm}$ inner diameter capillary was pulled but not to the point of pinch-off. The parallel plate-plate compression apparatus was constructed using a Mettler Toledo AT261 analytical balance recording the mass every second. The compression was controlled using a Newport Controller ESP300 operating a linear translation stage, Newport LTA-HL. To guarantee that both silicon wafers were parallel, the unpolished side of one silicon wafer was affixed to the balance pan using epoxy. The polished sides of the two wafers were then brought into physical contact. The translation stage was lowered and epoxy was used to affix the top silicon wafer to the stage; once cured the stage was raised and a single microbubble loaded between the plates. A compression test consisted of a single displacement step with a 15 min relaxation pause between steps to allow stresses to dissipate within both the permeable and impermeable microbubbles.

\section{AUTHOR INFORMATION}

\section{Corresponding Author}

*E-mail: wynter.duncanson@nu.edu.kz.

\section{Author Contributions}

${ }^{\S}$ W.J.D. and T.E.K. contributed equally.

\section{Notes}

The authors declare no competing financial interest.

\section{ACKNOWLEDGMENTS}

This work was supported by the National Science Foundation (NSF) (DMR-1310266), the Harvard MRSEC (DMR1420570), and the Advanced Energy Consortium, (BEG08027).

\section{REFERENCES}

(1) Calliada, F.; Campani, R.; Bottinelli, O.; Bozzini, A.; Sommaruga, M. G. Ultrasound contrast agents. Eur. J. Radiol. 1998, 27, S157-S160.

(2) Chen, R.; Dong, P.-F.; Xu, J.-H.; Wang, Y.-D.; Luo, G.-S. Controllable microfluidic production of gas-in-oil-in-water emulsions for hollow microspheres with thin polymer shells. Lab Chip 2012, 12, 3858.

(3) Tinkov, S.; Bekeredjian, R.; Winter, G.; Coester, C. Microbubbles as ultrasound triggered drug carriers. J. Pharm. Sci. 2009, 98, 19351961.

(4) Morris, R. H.; Bencsik, M.; Vangala, A. K.; Perrie, Y. Threedimensional fluid pressure mapping in porous media using magnetic resonance imaging with gas-filled liposomes. Magn. Reson. Imaging 2007, 25, 509-512.

(5) Vangala, A.; Morris, R.; Bencsik, M.; Perrie, Y. Preparation and characterization of gas-filled liposomes: Can they improve oil recovery? J. of Liposome Res. 2007, 17, 263-272.

(6) Chitnis, P. V., Koppolu, S., Mamou, J., Chlon, C., Ketterling, J. A. Influence of shell properties on high-frequency ultrasound imaging and drug delivery using polymer-shelled microbubbles Ultrason., Ferroelectr. Freq. Control 201360.

(7) Hettiarachchi, K.; Lee, A. P. Polymer-lipid microbubbles for biosensing and the formation of porous structures. J. Colloid Interface Sci. 2010, 344, 521-527.

(8) Lee, M. H.; Prasad, V.; Lee, D. Microfluidic fabrication of stable nanoparticle-shelled bubbles. Langmuir 2010, 26, 2227-2230.

(9) Talu, E.; Hettiarachchi, K.; Zhao, S.; Powell, R. L.; Lee, A. P.; Longo, M. L.; Dayton, P. A. Tailoring the size distribution of ultrasound contrast agents: Possible method for improving sensitivity in molecular imaging. Mol. Imaging. 2007, 6, 384-392.
(10) Wan, J.; Bick, A.; Sullivan, M.; Stone, H. A. Controllable microfluidic production of microbubbles in water-in-oil emulsions and the formation of porous microparticles. Adv. Mater. 2008, 20, 33143318.

(11) Abou-Saleh, R. H.; Peyman, S. A.; Critchley, K.; Evans, S. D.; Thomson, N. H. Nanomechanics of lipid encapsulated microbubbles with functional coatings. Langmuir 2013, 29, 4096-4103.

(12) Buchner Santos, E.; Morris, J. K.; Glynos, E.; Sboros, V.; Koutsos, V. Nanomechanical properties of phospholipid microbubbles. Langmuir 2012, 28, 5753-5760.

(13) Glynos, E.; Koutsos, V.; McDicken, W. N.; Moran, C. M.; Pye, S. D.; Ross, J. A.; Sboros, V. Nanomechanics of biocompatible hollow thin-shell polymer microspheres. Langmuir 2009, 25, 7514-7522.

(14) Fery, A.; Weinkamer, R. Mechanical properties of micro- and nanocapsules: Single-capsule measurements. Polymer 2007, 48, 72217235.

(15) Rachik, M.; Barthes-Biesel, D.; Carin, M.; Edwards-Levy, F. Identification of the elastic properties of an artificial capsule membrane with the compression test: Effect of thickness. J. Colloid Interface Sci. 2006, 301, 217-226.

(16) Mercade-Prieto, R.; Allen, R.; York, D.; Preece, J. A.; Goodwin, T. E.; Zhang, Z. Compression of elastic-perfectly plastic microcapsules using micromanipulation and finite element modelling: Determination of the yield stress. Chem. Eng. Sci. 2011, 66, 1835-1843.

(17) Utada, A. S.; Lorenceau, E.; Link, D. R.; Kaplan, P. D.; Stone, H. A.; Weitz, D. A. Monodisperse double emulsions generated from a microcapillary device. Science 2005, 308, 537-541.

(18) Hayward, R. C.; Utada, A. S.; Dan, N.; Weitz, D. A. Dewetting instability during the formation of polymersomes from blockcopolymer-stabilized double emulsions. Langmuir 2006, 22, 44574461.

(19) Lee, Y.-H.; Mei, F.; Bai, M.-Y.; Zhao, S.; Chen, D.-R. Release profile characteristics of biodegradable-polymer-coated drug particles fabricated by dual-capillary electrospray. J. Controlled Release 2010, $145,58-65$.

(20) Gref, R.; Domb, A.; Quellec, P.; Blunk, T.; Mueller, R.; Verbavatz, J.; Langer, R. The controlled intravenous delivery of drugs using PEG-coated sterically stabilized nanospheres. Adv. Drug Delivery Rev. 1995, 16, 215-233.

(21) Gref, R.; Quellec, P.; Sanchez, A.; Calvo, P.; Dellacherie, E.; Alonso, M. J. Development and characterization of CyA-loaded poly(lactic acid)-poly(ethylene glycol)PEG micro- and nanoparticles. Comparison with conventional PLA particulate carriers. Eur. J. Pharm. Biopharm. 2001, 51, 111-118.

(22) Yeoh, O. H.; Fleming, P. D. A new attempt to reconcile the statistical and phenomenological theories of rubber elasticity. J. Polym. Sci. B, Polym. Phys. 1997, 35, 1919-1931.

(23) Kim, S.-H.; Kim, J. W.; Cho, J.-C.; Weitz, D. A. Doubleemulsion drops with ultra-thin shells for capsule templates. Lab Chip 2011, 11, 3162 .

(24) Choi, K. M.; Rogers, J. A. A Photocurable poly(dimethylsiloxane) chemistry designed for soft lithographic molding and printing in the nanometer regime. J. Am. Chem. Soc. 2003, 125, 4060-4061. 